YITP-96-1

DPSU-96-1

January 1996

\title{
Reflection K-Matrices of the 19-Vertex Model and $X X Z$ Spin-1 Chain with General Boundary Terms
}

\author{
TAKeO INAMI ${ }^{a}$, SAtoru ODAKE ${ }^{b, \text { m }}$ and YAO-Zhong ZHANG ${ }^{a}$, \\ ${ }^{a}$ Yukawa Institute for Theoretical Physics \\ Kyoto University, Kyoto 606, Japan \\ ${ }^{b}$ Department of Physics, Faculty of Science \\ Shinshu University, Matsumoto 390, Japan
}

\begin{abstract}
We derive and classify all solutions of the boundary Yang-Baxter equation (or the reflection equation) for the 19-vertex model associated with $U_{q}\left(\widehat{s l_{2}}\right)$. Integrable $X X Z$ spin-1 chain hamiltonian with general boundary interactions is also obtained.
\end{abstract}

hep-th/9601049

\footnotetext{
${ }^{1}$ e-mail address : odake@yukawa.kyoto-u.ac.jp.

2 Address after June 6, 1996 : Department of Mathematics, University of Queenland, Brisbane, Qld 4072, Australia; e-mail address : yzzhang@yukawa.kyoto-u.ac.jp, yzz@maths.uq.oz.au.
} 


\section{Introduction}

Many problems in particle physics and condensed matter physics can be mapped to analogous problems in integrable quantum field theories and/or solvable lattice models in two dimensions by employing bold simplification or approximation. Understanding of integrable models has helped us gain insight into non-perturbative properties of fourdimensional quantum field theories, such as chiral symmetry breaking and confinement.

In some physical systems, effects of boundaries are very important and hence for physical application we have to extend integrable models to cases with boundaries. Methods for constructing integrable models with boundaries have recently been developed [1, 2, 3, 4. One systematic approach lies in finding the reflection matrix (or K-matrix) obeying the boundary Yang-Baxter equation (BYBE) (or reflection equation). Only few models with integrable boundary terms in full generality have so far been constructed: (super) sineGordon theory [3, 5], massive Thirring model [6], affine Toda field theories [7], $X X Z$ (and $X X X)$ [8, 9] and $X Y Z$ spin- $\frac{1}{2}$ chains [10].

Quantum spin chains have played important roles in many aspects of physics such as strongly correlated electron systems. Recently there has been experimental interest in quasi one-dimensional systems of molecules which mimic spin chains of higher spin $s$. To our knowledge, however, for spin chain with $s \geq 1$ only boundary terms corresponding to diagonal K-matrices are known (see, e.g., [11]).

In this paper we consider the 19-vertex model which is associated with the spin-1 representation of $U_{q}\left(\widehat{s l}_{2}\right)$. This model is the simplest extension of the six-vertex model. We construct and classify all reflection K-matrices of this model. From these K-matrices we derive the general integrable boundary terms for the $X X Z$ spin-1 chain. As a special case we reproduce the hamiltonian obtained in [11] by using the diagonal K-matrix.

\section{Nineteen-vertex Model}

The hamiltonian of the $X X Z$ spin-1 chain is derived from the transfer matrix of the 19 -vertex model. This vertex model is defined in terms of the Boltzmann weights given

by the R-matrix of spin-1 representation of $U_{q}\left(\widehat{s l_{2}}\right)$. It is the trigonometric solution of the Yang-Baxter equation (YBE):

$$
\left(1 \otimes \check{R}\left(u-u^{\prime}\right)\right)(\check{R}(u) \otimes 1)\left(1 \otimes \check{R}\left(u^{\prime}\right)\right)=\left(\check{R}\left(u^{\prime}\right) \otimes 1\right)(1 \otimes \check{R}(u))\left(\check{R}\left(u-u^{\prime}\right) \otimes 1\right)
$$


where $\check{R}(u)=P R(u)$ and $P$ is the transposition matrix: $P v_{1} \otimes v_{2}=v_{2} \otimes v_{1}$. $R(u)$ is a $9 \times 9$ matrix with 19 non-zero entries [12]:

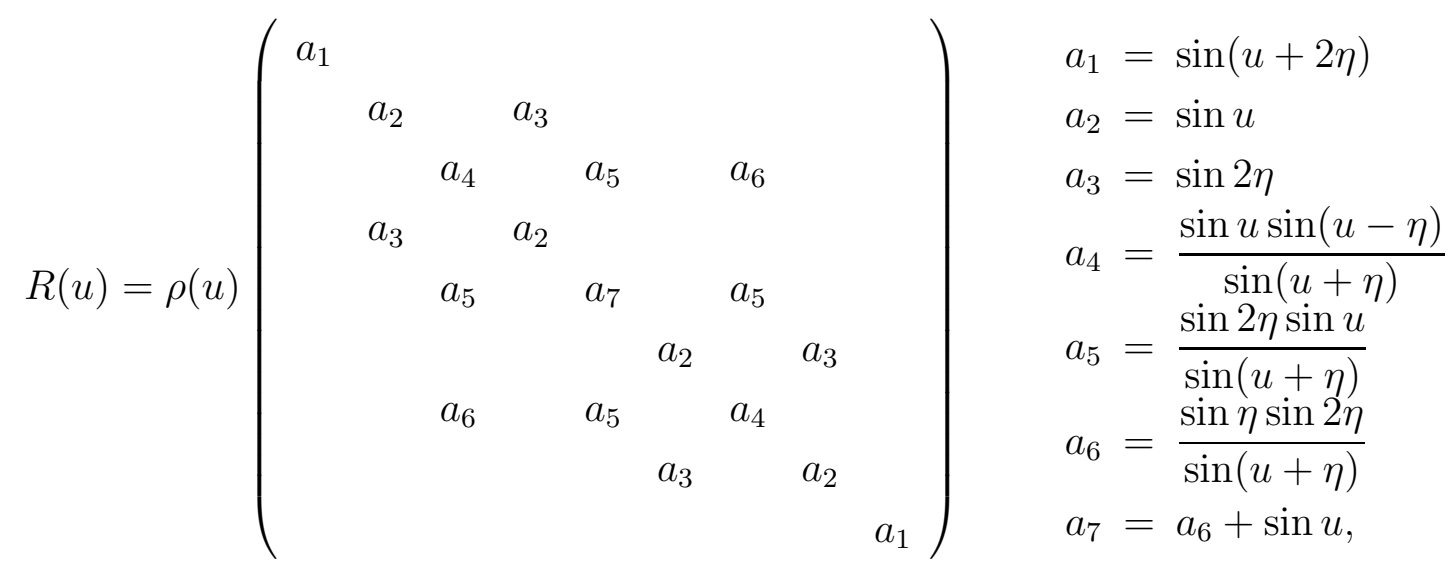

where $\rho(u) \not \equiv 0$ is an arbitrary function. If we choose $\rho(u)=\rho_{ \pm}(u)$,

$$
\rho_{ \pm}(u)=\frac{1}{\sin (2 \eta \pm u)}
$$

then $R$ satisfies

$$
\check{R}(u) \check{R}(-u)=1, \quad \check{R}(0)=1 .
$$

In the case of periodic boundary condition, the YBE implies a commuting family of transfer matrices and hence the integrability of the model.

\section{Reflection K-matrices}

We now consider the 19-vertex model with boundaries. As shown by Sklyanin [2], integrable models with boundaries can be constructed out of a pair of reflection K-matrices $K_{ \pm}(u)$ in addition to $R(u) . K_{+}(u)$ and $K_{-}(u)$ describe the effects of the presence of boundaries at the left and the right, respectively, and they both obey the BYBE.

Let us construct the K-matrix which satisfies the BYBE:

$$
R\left(u-u^{\prime}\right)(K(u) \otimes 1) R\left(u+u^{\prime}\right)\left(1 \otimes K\left(u^{\prime}\right)\right)=\left(1 \otimes K\left(u^{\prime}\right)\right) R\left(u+u^{\prime}\right)(K(u) \otimes 1) R\left(u-u^{\prime}\right) .
$$

In the present case, $R(u)$ is given in (2.2) and $K(u)$ can be parametrized as

$$
K(u)=\rho^{K}(u)\left(\begin{array}{ccc}
x_{1}(u) & y_{1}(u) & z(u) \\
\tilde{y}_{1}(u) & x_{2}(u) & y_{2}(u) \\
\tilde{z}(u) & \tilde{y}_{2}(u) & x_{3}(u)
\end{array}\right),
$$

where $\rho^{K}(u) \not \equiv 0$ is an arbitrary function.

The BYBE consists of 81 equations, many of which are dependent. To solve them, we proceed in a pragmatic fashion. The whole procedure is quite involved and requires a bit 
of computer work. Here we only present the final result. More details of the derivation are relegated to the Appendix.

We pick up a few easy-looking and independent equations. They are functional equations and can be reduced to first order differential equations or algebraic equations. The solutions to these equations contain several arbitrary parameters. We then substitute these partial solutions back into the BYBE, which gives a set of equations with the number of equations now less than 81 (since some equations are already satisfied). From this set of equations we pick up some simple-looking ones, solve them and determine the relations among the parameters. Repeating this process for a couple of times, we end up with the solutions with three arbitrary parameters satisfying all 81 functional equations. The solutions obtained in this way exhaust all solutions of (3.1) (for the 19-vertex model).

All solutions can be classified into the following three cases:

Class (A): $\sin 2 \eta \neq 0$.

$$
\begin{aligned}
& y_{1}(u)=\mu \sin \left(\zeta-\frac{\eta}{2}+u\right) \sin 2 u, \quad \tilde{y}_{1}(u)=\tilde{\mu} \sin \left(\zeta-\frac{\eta}{2}+u\right) \sin 2 u, \\
& y_{2}(u)=\mu \sin \left(\zeta+\frac{\eta}{2}-u\right) \sin 2 u, \quad \tilde{y}_{2}(u)=\tilde{\mu} \sin \left(\zeta+\frac{\eta}{2}-u\right) \sin 2 u, \\
& z(u)=\mu^{2} \frac{\sin (\eta-2 u)}{2 \cos \eta} \sin 2 u, \quad \tilde{z}(u)=\tilde{\mu}^{2} \frac{\sin (\eta-2 u)}{2 \cos \eta} \sin 2 u, \\
& x_{1}(u)=\sin \left(\frac{\eta}{2}+\zeta+u\right) \sin \left(\frac{\eta}{2}-\zeta-u\right)+\mu \tilde{\mu} \frac{\sin (\eta-2 u)}{2 \cos \eta} \sin \eta, \\
& x_{2}(u)=\sin \left(\frac{\eta}{2}+\zeta-u\right) \sin \left(\frac{\eta}{2}-\zeta-u\right)+\mu \tilde{\mu} \frac{\sin (\eta-2 u)}{2 \cos \eta} \sin (\eta+2 u), \\
& x_{3}(u)=\sin \left(\frac{\eta}{2}+\zeta-u\right) \sin \left(\frac{\eta}{2}-\zeta+u\right)+\mu \tilde{\mu} \frac{\sin (\eta-2 u)}{2 \cos \eta} \sin \eta,
\end{aligned}
$$

where $\zeta, \mu, \tilde{\mu}$, are arbitrary constants. If we choose $\rho^{K}(u)=\rho_{ \pm}^{K}(u)$ with

$$
\rho_{ \pm}^{K}(u)=\frac{1}{\sin \left(\frac{\eta}{2}+\zeta \pm u\right) \sin \left(\frac{\eta}{2}-\zeta \pm u\right)+\mu \tilde{\mu} \sin ^{2}(\eta \pm 2 u) /(2 \cos \eta)}
$$

then $K$ satisfies

$$
K(u) K(-u)=1, \quad K(0)=1 .
$$

Class (B): $\sin 2 \eta \neq 0$ and $\sin 4 \eta=0$.

For this case we have three solutions:

$$
\begin{aligned}
x_{1}(u) & =\sin \left(\frac{\eta}{2}+\zeta+u\right) \sin \left(\frac{\eta}{2}-\zeta-u\right), x_{2}(u)=\sin \left(\frac{\eta}{2}+\zeta-u\right) \sin \left(\frac{\eta}{2}-\zeta-u\right), \\
x_{3}(u) & =\sin \left(\frac{\eta}{2}+\zeta-u\right) \sin \left(\frac{\eta}{2}-\zeta+u\right), y_{1}(u)=y_{2}(u)=\tilde{y}_{1}(u)=\tilde{y}_{2}(u)=0, \\
z(u) & =\nu \sin 2 u, \tilde{z}(u)=0, \quad \text { or } \quad z(u)=0, \tilde{z}(u)=\nu \sin 2 u,
\end{aligned}
$$


with $\nu \neq 0$ and $\zeta(\sin 2 \zeta \neq 0)$ being arbitrary constants, and

$$
\begin{aligned}
& y_{1}(u)=y_{2}(u)=\tilde{y}_{1}(u)=\tilde{y}_{2}(u)=x_{2}(u)=0, \\
& x_{1}(u)=1, x_{3}(u)=-1, z(u)=\nu, \tilde{z}(u)=-\frac{1}{\nu},
\end{aligned}
$$

where $\nu \neq 0$ is an arbitrary constant.

If we take $\rho^{K}(u)=1 /\left(\sin \left(\frac{\eta}{2}+\zeta \pm u\right) \sin \left(\frac{\eta}{2}-\zeta \pm u\right)\right.$ ) (four choices), then K-matrices (3.6) satisfy (3.5). However, K-matrix (3.7) does not satisfy (3.5).

Class (C): $\sin 2 \eta=0$.

For $\eta=0, \pi$, there are no restriction for $K$. For $\eta=\frac{1}{2} \pi, \frac{3}{2} \pi$, we have $y_{1}=y_{2}=\tilde{y}_{1}=$ $\tilde{y}_{2}=0$ and other components are arbitrary.

\section{$4 X X Z$ Spin-1 Chain with General Boundary}

In this section we consider the K-matrix (3.3), $K(u)=K(u ; \zeta, \mu, \tilde{\mu})$, and take $\rho(u)=$ $\rho_{ \pm}(u)$ and $\rho^{K}(u)=\rho_{ \pm}^{K}(u) . K_{ \pm}(u)$ are defined as $K_{-}(u)=K\left(u ; \zeta_{-}, \mu_{-}, \tilde{\mu}_{-}\right), K_{+}(u)=$ ${ }^{t} K\left(-u-\eta ;-\zeta_{+}, \tilde{\mu}_{+}, \mu_{+}\right)$.

$\mathrm{R}$ - and $\mathrm{K}$-matrices induce the hamiltonian of integrable spin-1 $X X Z$ open chain with $N$ sites,

$$
H=\sum_{n=1}^{N-1} H_{n, n+1}+\left.\frac{1}{2} \frac{d}{d u} K_{-, 1}(u)\right|_{u=0}+\frac{\operatorname{tr}_{0} K_{+, 0}(0) H_{N, 0}}{\operatorname{tr} K_{+}(0)},
$$

where the two-site hamiltonian is given by

$$
H_{n, n+1}=\left.\frac{d}{d u} \check{R}_{n, n+1}(u)\right|_{u=0}
$$

Here suffices of $\check{R}$ and $K_{ \pm}$etc. indicate the sites on which the operators act. We remark that the terms in $H$ which are not proportional to identity do not depend on the four choices $\left(\rho_{ \pm}, \rho_{ \pm}^{K}\right)$. From $(2.2),(2.3),(3.3),(3.4)$, we obtain

$$
\begin{aligned}
& H=\frac{1}{\sin 2 \eta} \sum_{n=1}^{N-1}\left(\vec{s}_{n} \cdot \vec{s}_{n+1}-\left(\vec{s}_{n} \cdot \vec{s}_{n+1}\right)^{2}+(1-\cos \eta)\left\{s_{n}^{3} s_{n+1}^{3}, s_{n}^{+} s_{n+1}^{-}+s_{n}^{-} s_{n+1}^{+}\right\}\right. \\
& \left.\quad-(1-\cos 2 \eta)\left(s_{n}^{3} s_{n+1}^{3}-\left(s_{n}^{3}\right)^{2}\left(s_{n+1}^{3}\right)^{2}+\left(s_{n}^{3}\right)^{2}+\left(s_{n+1}^{3}\right)^{2}\right)\right) \\
& +\frac{1}{2 \sin \left(\frac{\eta}{2}+\zeta_{-}\right) \sin \left(\frac{\eta}{2}-\zeta_{-}\right)+\mu_{-} \tilde{\mu}_{-} \sin \eta \tan \eta} \\
& \quad \times\left(\left(1-\mu_{-} \tilde{\mu}_{-}\right) \sin \eta s_{1}^{3} s_{1}^{3}-\sin 2 \zeta_{-} s_{1}^{3}+\frac{1}{2} \tan \eta\left(\mu_{-}^{2} s_{1}^{+} s_{1}^{+}+\tilde{\mu}_{-}^{2} s_{1}^{-} s_{1}^{-}\right)\right. \\
& \left.\quad-\sqrt{2} \sin \left(\frac{\eta}{2}+\zeta_{-}\right)\left(\mu_{-} s_{1}^{+} s_{1}^{3}+\tilde{\mu}_{-} s_{1}^{3} s_{1}^{-}\right)-\sqrt{2} \sin \left(\frac{\eta}{2}-\zeta_{-}\right)\left(\mu_{-} s_{1}^{3} s_{1}^{+}+\tilde{\mu}_{-} s_{1}^{-} s_{1}^{3}\right)\right)
\end{aligned}
$$




$$
\begin{aligned}
& +\frac{1}{2 \sin \left(\frac{\eta}{2}+\zeta_{+}\right) \sin \left(\frac{\eta}{2}-\zeta_{+}\right)+\mu_{+} \tilde{\mu}_{+} \sin \eta \tan \eta} \\
& \quad \times\left(\left(1-\mu_{+} \tilde{\mu}_{+}\right) \sin \eta s_{N}^{3} s_{N}^{3}-\sin 2 \zeta_{+} s_{N}^{3}+\frac{1}{2} \tan \eta\left(\mu_{+}^{2} s_{N}^{+} s_{N}^{+}+\tilde{\mu}_{+}^{2} s_{N}^{-} s_{N}^{-}\right)\right. \\
& \left.\quad-\sqrt{2} \sin \left(\frac{\eta}{2}+\zeta_{+}\right)\left(\mu_{+} s_{N}^{+} s_{N}^{3}+\tilde{\mu}_{+} s_{N}^{3} s_{N}^{-}\right)-\sqrt{2} \sin \left(\frac{\eta}{2}-\zeta_{+}\right)\left(\mu_{+} s_{N}^{3} s_{N}^{+}+\tilde{\mu}_{+} s_{N}^{-} s_{N}^{3}\right)\right) \\
& +(\text { constant }) \cdot \mathrm{id},
\end{aligned}
$$

where the spin-1 operator $s^{3}, s^{ \pm}\left(=s^{1} \pm i s^{2}\right)$ is given by

$$
s^{3}=\left(\begin{array}{ccc}
1 & 0 & 0 \\
0 & 0 & 0 \\
0 & 0 & -1
\end{array}\right), s^{+}=\sqrt{2}\left(\begin{array}{ccc}
0 & 1 & 0 \\
0 & 0 & 1 \\
0 & 0 & 0
\end{array}\right), s^{-}=\sqrt{2}\left(\begin{array}{ccc}
0 & 0 & 0 \\
1 & 0 & 0 \\
0 & 1 & 0
\end{array}\right) \text {. }
$$

In the special case, $\mu_{ \pm}=\tilde{\mu}_{ \pm}=0$, (4.3) reduces to the hamiltonian obtained in [11 by using the diagonal K-matrix.

\section{Fusion Procedure}

The R-matrix (2.2) of the 19-vertex model was obtained by directly solving the YBE in [12]. It can also be constructed out of the R-matrix of the six-vertex model by using the fusion method [13].

It was suggested that the fusion method can also be used to construct K-matrices of vertex models corresponding to higher spins out of the K-matrix of the six-vertex model [11. In this section we apply this fusion method to the present case and compare the result derived this way with that obtained above by directly solving the BYBE.

The R-matrix associated with the spin- $\frac{1}{2}$ representation of $U_{q}\left(\widehat{s l_{2}}\right)$ reads,

$$
R^{\left(\frac{1}{2}, \frac{1}{2}\right)}(u)=\rho_{\frac{1}{2}}(u)\left(\begin{array}{cccc}
\sin (\eta+u) & 0 & 0 & 0 \\
0 & \sin u & \sin \eta & 0 \\
0 & \sin \eta & \sin u & 0 \\
0 & 0 & 0 & \sin (\eta+u)
\end{array}\right)
$$

with $\rho_{\frac{1}{2}}(u) \not \equiv 0$ being an arbitrary function. The corresponding reflection K-matrix has the form [8]

$$
K^{\left(\frac{1}{2}\right)}(u)=\rho_{\frac{1}{2}}^{K}(u)\left(\begin{array}{cc}
\sin (\xi+u) & \nu \sin 2 u \\
\tilde{\nu} \sin 2 u & \sin (\xi-u)
\end{array}\right),
$$

where $\xi, \nu, \tilde{\nu}$ are constant parameters and $\rho_{\frac{1}{2}}^{K}(u) \not \equiv 0$ is an arbitrary overall function.

According to [11] the K-matrix for the 19-vertex model is given by the following fusion equation:

$$
K(u)=f(u) U P_{+}\left(K^{\left(\frac{1}{2}\right)}\left(u-\frac{\eta}{2}\right) \otimes 1\right) R^{\left(\frac{1}{2}, \frac{1}{2}\right)}(2 u)\left(1 \otimes K^{\left(\frac{1}{2}\right)}\left(u+\frac{\eta}{2}\right)\right) P_{+} U^{-1},
$$


where $P_{+}$is the projection onto the spin-1 component and $U$ is a basis-changing matrix; explicitly,

$$
P_{+}=\frac{1}{2}(1+P)=\left(\begin{array}{cccc}
1 & 0 & 0 & 0 \\
0 & \frac{1}{2} & \frac{1}{2} & 0 \\
0 & \frac{1}{2} & \frac{1}{2} & 0 \\
0 & 0 & 0 & 1
\end{array}\right), \quad U=\left(\begin{array}{cccc}
1 & 0 & 0 & 0 \\
0 & \alpha & \alpha & 0 \\
0 & 0 & 0 & 1 \\
0 & \alpha & -\alpha & 0
\end{array}\right)
$$

It turns out that the K-matrix $K(u)$ from (5.3) agrees with one of the solutions in section 3 , eq.(3.3), if one makes the following identification:

$$
\begin{aligned}
& f(u)=-\frac{1}{\sin (\eta+2 u)} \frac{\rho^{K}(u)}{\rho_{\frac{1}{2}}^{K}\left(u-\frac{\eta}{2}\right) \rho_{\frac{1}{2}}^{K}\left(u+\frac{\eta}{2}\right) \rho_{\frac{1}{2}}(2 u)}, \\
& \xi=\zeta, \nu=\frac{\mu}{\sqrt{2 \cos \eta}}, \tilde{\nu}=\frac{\tilde{\mu}}{\sqrt{2 \cos \eta}}, \alpha=-\frac{\mu}{2 \nu} .
\end{aligned}
$$

It seems that the solutions in Class (B) can not be obtained from this fusion procedure.

Acknowledgments: This work is supported in part by the Grant-in-Aid for Scientific Research from the Ministry of Education, Science and Culture of Japan, and also by Japan-Korea exchange program of JSPS. Y.Z.Z. is financially supported by JSPS.

\section{A Derivation of the Reflection K-matrices}

In this Appendix we sketch how (3.3), (3.6) and (3.7) are obtained and show that there is no other nontrivial solution (Class (C) is trivial!). Our derivation is based on a careful case by case study. We sometimes omit explicit expressions when they may be lengthy. First we remark that one can check (3.3), (3.6) and (3.7) by direct substitution. Let us denote $(i, j)$ component of (3.1) as $\mathrm{Eq}[i, j]$. Note that $\mathrm{Eq}[10-i, 10-j]$ can be obtained from $\mathrm{Eq}[i, j]$ by interchanging $x_{1} \leftrightarrow x_{3}, y_{1} \leftrightarrow \tilde{y}_{2}, y_{2} \leftrightarrow \tilde{y}_{1}, z \leftrightarrow \tilde{z}$. In the following we assume $\sin 2 \eta \neq 0$ (as mentioned above, the $\sin 2 \eta=0$ case is trivial).

I. From $\mathrm{Eq}[2,8]$, we have

$$
\sin \left(u-u^{\prime}\right)\left(y_{1}(u) y_{1}\left(u^{\prime}\right)-y_{2}(u) y_{2}\left(u^{\prime}\right)\right)=\sin \left(\eta-u-u^{\prime}\right)\left(y_{1}\left(u^{\prime}\right) y_{2}(u)-y_{1}(u) y_{2}\left(u^{\prime}\right)\right) .
$$

Dividing this by $\sin \left(u-u^{\prime}\right) \sin \left(\eta-u-u^{\prime}\right) y_{2}(u) y_{2}\left(u^{\prime}\right)$ and taking $u^{\prime} \rightarrow u$ limit, we obtain the differential equation

$$
\frac{d}{d u}\left(\frac{y_{1}}{y_{2}}\right)=\frac{1-\left(\frac{y_{1}}{y_{2}}\right)^{2}}{\sin (\eta-2 u)}
$$


Solving this, we get

$$
\frac{y_{1}(u)}{y_{2}(u)}=\frac{\sin \left(\zeta-\frac{\eta}{2}+u\right)}{\sin \left(\zeta+\frac{\eta}{2}-u\right)},
$$

where $\zeta$ is a constant. We can check that this relation satisfies (A.1).

$\mathrm{Eq}[2,9]$ gives rise to

$$
\sin \left(\eta-u-u^{\prime}\right) y_{2}(u) z\left(u^{\prime}\right)-\sin \left(\eta-2 u^{\prime}\right) y_{2}\left(u^{\prime}\right) z(u)=\sin \left(u-u^{\prime}\right) y_{1}(u) z\left(u^{\prime}\right) .
$$

By differentiating this linear equation with respect to $u^{\prime}$ and taking $u^{\prime} \rightarrow u$ limit, we have

$$
y_{1}=-\cos (\eta-2 u) y_{2}+\sin (\eta-2 u) y_{2}\left(\frac{1}{y_{2}} \frac{d y_{2}}{d u}-\frac{1}{z} \frac{d z}{d u}\right) .
$$

Solving this differential equation with the help of (A.2), we obtain

$$
\frac{y_{2}(u)}{z(u)}=\frac{\sin \left(\zeta+\frac{\eta}{2}-u\right)}{\sin (\eta-2 u)} \times(\text { constant })
$$

Again we can check that this relation satisfies (A.3). Up to now, we have assumed $y_{2}$ and $z$ are not zero. Taking into account the fact that they may be zero, we obtain the following result from (A.1) and (A.3),

$$
y_{1}(u)=\mu \sin \left(\zeta-\frac{\eta}{2}+u\right) g(u), y_{2}(u)=\mu \sin \left(\zeta+\frac{\eta}{2}-u\right) g(u), z(u)=\nu \sin (\eta-2 u) g(u),
$$

where $\zeta, \mu, \nu$ are arbitrary constants and $g(u) \not \equiv 0$ is an arbitrary function.

Similarly, by Eq[8,2] and $\mathrm{Eq}[9,2]$,

$$
\tilde{y}_{1}(u)=\tilde{\mu}^{\prime} \sin \left(\tilde{\zeta}-\frac{\eta}{2}+u\right) \tilde{g}(u), \tilde{y}_{2}(u)=\tilde{\mu}^{\prime} \sin \left(\tilde{\zeta}+\frac{\eta}{2}-u\right) \tilde{g}(u), \tilde{z}(u)=\tilde{\nu}^{\prime} \sin (\eta-2 u) \tilde{g}(u),
$$

where $\tilde{\zeta}, \tilde{\mu}^{\prime}, \tilde{\nu}^{\prime}$ are arbitrary constants and $\tilde{g}(u) \not \equiv 0$ is an arbitrary function.

From $\mathrm{Eq}[1,1]$ and $\mathrm{Eq}[9,9]$, we have

$$
\begin{aligned}
& \sin \left(\eta+u+u^{\prime}\right)\left(\tilde{y}_{1}\left(u^{\prime}\right) y_{1}(u)-\tilde{y}_{1}(u) y_{1}\left(u^{\prime}\right)\right)=\sin \eta\left(z\left(u^{\prime}\right) \tilde{z}(u)-z(u) \tilde{z}\left(u^{\prime}\right)\right), \\
& \sin \left(\eta+u+u^{\prime}\right)\left(\tilde{y}_{2}\left(u^{\prime}\right) y_{2}(u)-\tilde{y}_{2}(u) y_{2}\left(u^{\prime}\right)\right)=\sin \eta\left(z\left(u^{\prime}\right) \tilde{z}(u)-z(u) \tilde{z}\left(u^{\prime}\right)\right) .
\end{aligned}
$$

Using (A.5) and (A.6), we arrive at the following three partial results:

$$
\begin{aligned}
& \text { (1) }\left\{\begin{aligned}
y_{1}(u) & =\mu \sin \left(\zeta-\frac{\eta}{2}+u\right) g(u), & \tilde{y}_{1}(u) & =\tilde{\mu} \sin \left(\zeta-\frac{\eta}{2}+u\right) g(u), \\
y_{2}(u) & =\mu \sin \left(\zeta+\frac{\eta}{2}-u\right) g(u), & \tilde{y}_{2}(u) & =\tilde{\mu} \sin \left(\zeta+\frac{\eta}{2}-u\right) g(u), \\
z(u) & =\nu \sin (\eta-2 u) g(u), & \tilde{z}(u) & =\tilde{\nu} \sin (\eta-2 u) g(u),
\end{aligned}\right. \\
& (2)\left\{\begin{aligned}
y_{1}(u) & =\sin \left(\zeta-\frac{\eta}{2}+u\right) g(u), & \tilde{y}_{1}(u) & =0, \\
y_{2}(u) & =\sin \left(\zeta+\frac{\eta}{2}-u\right) g(u), & \tilde{y}_{2}(u) & =0, \\
z(u) & =0, & \tilde{z}(u) & =\sin (\eta-2 u) \tilde{g}(u),
\end{aligned}\right. \\
& \text { (3) }\left\{\begin{aligned}
y_{1}(u) & =0, & \tilde{y}_{1}(u) & =\sin \left(\tilde{\zeta}-\frac{\eta}{2}+u\right) \tilde{g}(u), \\
y_{2}(u) & =0, & \tilde{y}_{2}(u) & =\sin \left(\tilde{\zeta}+\frac{\eta}{2}-u\right) \tilde{g}(u), \\
z(u) & =\sin (\eta-2 u) g(u), & \tilde{z}(u) & =0,
\end{aligned}\right.
\end{aligned}
$$


where $\zeta, \tilde{\zeta}, \mu, \tilde{\mu}, \nu, \tilde{\nu}$ are arbitrary constants and $g(u)$ and $\tilde{g}(u)$ are arbitrary functions $(g, \tilde{g} \not \equiv 0, g / \tilde{g} \not \equiv$ constant $)$.

II. First let us consider case (2). $\mathrm{Eq}[4,1]$ and $\mathrm{Eq}[2,1]$ can be written as the following form respectively

$$
\begin{aligned}
& A_{1}\left(u, u^{\prime}\right) g(u) \tilde{g}\left(u^{\prime}\right)+A_{2}\left(u, u^{\prime}\right) g\left(u^{\prime}\right) \tilde{g}(u)=0, \\
& A_{3}\left(u, u^{\prime}\right) g(u) \tilde{g}\left(u^{\prime}\right)+A_{4}\left(u, u^{\prime}\right) g\left(u^{\prime}\right) \tilde{g}(u)=0,
\end{aligned}
$$

where $A_{i}$ 's are known functions of $u$ and $u^{\prime}$ with their explicit expressions omitted. Since $g(u) \tilde{g}\left(u^{\prime}\right) \not \equiv 0$, we have $\Delta\left(u, u^{\prime}\right) \equiv A_{1} A_{4}-A_{2} A_{3}=0 . \Delta(0, \eta)=0$ means $\sin 2 \zeta=0$. With this $\zeta$, however, $\Delta(u,-u)$ does not vanish, which implies the above two equations are contradictory each other. Therefore case (2) is not a solution.

Similarly, from Eq[1,4] and $\mathrm{Eq}[1,2]$, we can show that case (3) is not a solution.

III. In the following we consider case (1). Eq[1,4] is linear in $x_{1}(u), x_{1}\left(u^{\prime}\right), x_{2}(u), x_{2}\left(u^{\prime}\right)$. There are two cases to be considered: $\mu=0$ and $\mu \neq 0$. For the former case, Eq[1,4] is satisfied if and only if $\tilde{\mu} \nu=0$. For the latter case, the coefficient of $x_{2}\left(u^{\prime}\right)$ vanishes and $x_{2}(u)$ can be expressed by $x_{1}(u)$ and $x_{1}\left(u^{\prime}\right)$. Of course this expression must be independent on $u^{\prime}$. Similar results can be obtained for $\mathrm{Eq}[4,1]$. Combining these results, we derive

$$
\begin{aligned}
\text { (i) } & \mu=0, \tilde{\mu}=0 \\
\text { (ii) } & \mu=0, \tilde{\mu} \neq 0 \quad(\Rightarrow \nu=0) \\
\text { (iii) } & \mu \neq 0, \tilde{\mu}=0 \quad(\Rightarrow \tilde{\nu}=0) \\
\text { (iv-a) } & \mu \neq 0, \tilde{\mu} \neq 0, \nu=0 \quad(\Rightarrow \tilde{\nu}=0) \\
\text { (iv-b) } & \mu \neq 0, \tilde{\mu} \neq 0, \nu \neq 0 \quad\left(\Rightarrow \tilde{\nu}=\left(\frac{\tilde{\mu}}{\mu}\right)^{2} \nu, \sin (\eta \mp 2 \zeta) \neq 0\right)
\end{aligned}
$$

(the condition $\sin (\eta+2 \zeta) \neq 0$ is obtained below). For (i) there is no restriction on $x_{1}, x_{2}$. For other cases,

$$
\begin{aligned}
& x_{1}(u)=\left(\frac{\lambda_{1}}{\sin u}+\frac{\lambda_{2}}{\cos u}\right) \sin \left(\frac{\eta}{2}-\zeta-u\right) g(u)+\delta \frac{\tilde{\mu} \nu}{\mu} \frac{\sin \eta \sin 2 \zeta}{\sin (\eta-2 \zeta)} g(u), \\
& x_{2}(u)=\left(\frac{\lambda_{1}}{\sin u}-\frac{\lambda_{2}}{\cos u}\right) \sin \left(\frac{\eta}{2}-\zeta-u\right) g(u)+\delta \frac{\tilde{\mu} \nu}{\mu}\left(\frac{\sin ^{2} \eta}{\sin (\eta-2 \zeta)}-\sin 2 u\right) g(u),
\end{aligned}
$$

where $\lambda_{1}, \lambda_{2}$ are arbitrary constants, and $\delta=1$ for case (iv-b) and $\delta=0$ for other cases.

Similarly from Eq[9,6] and $\mathrm{Eq}[6,9]$ we obtain $x_{3}$ and $x_{2}$ (and the condition $\sin (\eta+2 \zeta) \neq$ 0 in the case (iv-b) above): for (i) there is no restriction on $x_{3}, x_{2}$, and for other cases,

$$
\begin{aligned}
& x_{3}(u)=\left(\frac{\lambda_{3}}{\sin u}+\frac{\lambda_{4}}{\cos u}\right) \sin \left(\frac{\eta}{2}+\zeta-u\right) g(u)-\delta \frac{\tilde{\mu} \nu}{\mu} \frac{\sin \eta \sin 2 \zeta}{\sin (\eta+2 \zeta)} g(u), \\
& x_{2}(u)=\left(\frac{\lambda_{3}}{\sin u}-\frac{\lambda_{4}}{\cos u}\right) \sin \left(\frac{\eta}{2}+\zeta-u\right) g(u)+\delta \frac{\tilde{\mu} \nu}{\mu}\left(\frac{\sin ^{2} \eta}{\sin (\eta+2 \zeta)}-\sin 2 u\right) g(u),
\end{aligned}
$$


where $\lambda_{3}, \lambda_{4}$ are arbitrary constants, and $\delta=1$ for case (iv-b) and $\delta=0$ for other cases.

Combining (A.13) and (A.14) (for example taking the limit $u \rightarrow 0, \frac{\pi}{2}$ ), we get

$$
\begin{aligned}
x_{1}(u)= & \frac{2 \lambda}{\sin 2 u} \sin \left(\frac{\eta}{2}+\zeta+u\right) \sin \left(\frac{\eta}{2}-\zeta-u\right) g(u) \\
& +\delta \frac{\tilde{\mu} \nu}{\mu} \frac{\sin \eta}{\sin (\eta-2 \zeta)}\left(\sin 2 \zeta-\frac{\sin \left(\frac{\eta}{2}-\zeta-u\right) \sin 2 \eta}{2 \cos u \sin \left(\frac{\eta}{2}+\zeta\right)}\right) g(u), \\
x_{2}(u)= & \frac{2 \lambda}{\sin 2 u} \sin \left(\frac{\eta}{2}+\zeta-u\right) \sin \left(\frac{\eta}{2}-\zeta-u\right) g(u) \\
& +\delta \frac{\tilde{\mu} \nu}{\mu} \frac{\sin \eta}{\sin (\eta-2 \zeta)}\left(\sin \zeta+\frac{\sin \left(\frac{\eta}{2}-\zeta-u\right) \sin 2 \eta}{2 \cos u \sin \left(\frac{\eta}{2}+\zeta\right)}\right) g(u)-\delta \frac{\tilde{\mu} \nu}{\mu} \sin 2 u g(u), \\
x_{3}(u)= & \frac{2 \lambda}{\sin 2 u} \sin \left(\frac{\eta}{2}-\zeta+u\right) \sin \left(\frac{\eta}{2}+\zeta-u\right) g(u) \\
& +\delta \frac{\tilde{\mu} \nu}{\mu} \frac{\sin \eta}{\sin (\eta+2 \zeta)}\left(-\sin 2 \zeta-\frac{\sin \left(\frac{\eta}{2}+\zeta-u\right) \sin 2 \eta}{2 \cos u \sin \left(\frac{\eta}{2}-\zeta\right)}\right) g(u),
\end{aligned}
$$

where $\delta=1$ for case (iv-b) and $\delta=0$ for cases (ii),(iii),(iv-a). For case (i) there are no restriction for $x_{1}, x_{2}, x_{3}$.

IV. Case (i).

Let $X_{i}(u)=x_{i}(u) /(\sin (\eta-2 u) g(u))$. Eq[4,6] and $\operatorname{Eq}[6,4]$ lead to

$$
\begin{aligned}
& \nu\left(X_{1}(u)-X_{3}(u)-\left(X_{1}\left(u^{\prime}\right)-X_{3}\left(u^{\prime}\right)\right)\right)=0, \\
& \tilde{\nu}\left(X_{1}(u)-X_{3}(u)-\left(X_{1}\left(u^{\prime}\right)-X_{3}\left(u^{\prime}\right)\right)\right)=0,
\end{aligned}
$$

and $\mathrm{Eq}[2,4]$ and $\mathrm{Eq}[6,8]$ to

$$
\begin{aligned}
& \frac{X_{1}(u) X_{1}\left(u^{\prime}\right)-X_{2}(u) X_{2}\left(u^{\prime}\right)+\nu \tilde{\nu}}{\sin \left(u+u^{\prime}\right)}=\frac{X_{1}(u) X_{2}\left(u^{\prime}\right)-X_{1}\left(u^{\prime}\right) X_{2}(u)}{\sin \left(u-u^{\prime}\right)}, \\
& \frac{X_{3}(u) X_{3}\left(u^{\prime}\right)-X_{2}(u) X_{2}\left(u^{\prime}\right)+\nu \tilde{\nu}}{\sin \left(u+u^{\prime}\right)}=\frac{X_{3}(u) X_{2}\left(u^{\prime}\right)-X_{3}\left(u^{\prime}\right) X_{2}(u)}{\sin \left(u-u^{\prime}\right)} .
\end{aligned}
$$

We have two choices (a) $\nu \tilde{\nu}=0$, (b) $\nu \tilde{\nu} \neq 0$.

IV.1. Case (a).

Through a similar calculation as for (A.1), it follows from (A.18) and (A.19) that for $x_{2} \equiv 0$, we have $x_{1}(u)=x_{2}(u)=x_{3}(u)=0$, and for $x_{2} \not \equiv 0$, we get

$$
\begin{aligned}
& x_{1}(u)=\sin \left(c_{1}-u\right) \sin \left(c_{2}+u\right) f(u), \\
& x_{2}(u)=\sin \left(c_{1}+u\right) \sin \left(c_{2}+u\right) f(u), \\
& x_{3}(u)=\sin \left(c_{1}+u\right) \sin \left(c_{2}-u\right) f(u),
\end{aligned}
$$

where $c_{1}, c_{2}$ are arbitrary constants and $f(u) \not \equiv 0$ is an arbitrary function. For the former case, we can check that they give the following two solutions of (3.1):

$$
\begin{aligned}
& z(u)=h(u), x_{1}=x_{2}=x_{3}=y_{1}=y_{2}=\tilde{y}_{1}=\tilde{y}_{2}=\tilde{z}=0, \\
& \tilde{z}(u)=h(u), x_{1}=x_{2}=x_{3}=y_{1}=y_{2}=\tilde{y}_{1}=\tilde{y}_{2}=z=0,
\end{aligned}
$$


where $h(u) \not \equiv 0$ is an arbitrary function. For the latter case, Eq[3,5] implies $\sin \left(c_{1}+\right.$ $\left.c_{2}+\eta\right)=0$. Since $x_{i}$ 's are invariant under $c_{2} \rightarrow c_{2}+\pi$ and $f(u) \rightarrow-f(u)$, we can take $c_{1}+c_{2}+\eta=0$. Therefore we have

$$
\begin{aligned}
& x_{1}(u)=\sin \left(\frac{\eta}{2}+\zeta+u\right) \sin \left(\frac{\eta}{2}-\zeta-u\right) f(u), \\
& x_{2}(u)=\sin \left(\frac{\eta}{2}+\zeta-u\right) \sin \left(\frac{\eta}{2}-\zeta-u\right) f(u), \\
& x_{3}(u)=\sin \left(\frac{\eta}{2}+\zeta-u\right) \sin \left(\frac{\eta}{2}-\zeta+u\right) f(u),
\end{aligned}
$$

where $\zeta$ is an arbitrary constant. The following three cases are possible:

$$
\begin{array}{ll}
\text { (a1) } & \nu=\tilde{\nu}=0, \\
\text { (a2) } & \nu \neq 0, \tilde{\nu}=0, \\
\text { (a3) } & \nu=0, \tilde{\nu} \neq 0 .
\end{array}
$$

- Case (a1). We can check that (3.1) is satisfied. This is a diagonal solution,

$$
\begin{aligned}
& y_{1}(u)=y_{2}(u)=\tilde{y}_{1}(u)=\tilde{y}_{2}(u)=z(u)=\tilde{z}(u)=0 \\
& x_{1}(u)=\sin \left(\frac{\eta}{2}+\zeta+u\right) \sin \left(\frac{\eta}{2}-\zeta-u\right) f(u) \\
& x_{2}(u)=\sin \left(\frac{\eta}{2}+\zeta-u\right) \sin \left(\frac{\eta}{2}-\zeta-u\right) f(u) \\
& x_{3}(u)=\sin \left(\frac{\eta}{2}+\zeta-u\right) \sin \left(\frac{\eta}{2}-\zeta+u\right) f(u)
\end{aligned}
$$

where $\zeta$ is an arbitrary constant and $f(u) \not \equiv 0$ is an arbitrary function.

- Case (a2). From (A.16) we have $x_{1}(u)-x_{3}(u)=\sigma \sin (\eta-2 u) g(u)$, where $\sigma$ is a constant. From (A.23) we get $-\sin 2 \zeta \sin 2 u f(u)=\sigma \sin (\eta-2 u) g(u)$. There are two choices:

$$
\begin{array}{ll}
(\mathrm{a} 2-1) & \sin 2 \zeta \neq 0(\Rightarrow \sigma \neq 0), \\
(\mathrm{a} 2-2) & \sin 2 \zeta=0(\Rightarrow \sigma=0) .
\end{array}
$$

For the case (a2-1), Eq[1,3] implies $\sin 4 \eta=0$ and then we can check that (3.1) is satisfied. Therefore we get a solution (note: $\sin 4 \eta=0$ ),

$$
\begin{aligned}
z(u) & =\nu \sin 2 u h(u), \quad y_{1}(u)=y_{2}(u)=\tilde{y}_{1}(u)=\tilde{y}_{2}(u)=\tilde{z}(u)=0, \\
x_{1}(u) & =\sin \left(\frac{\eta}{2}+\zeta+u\right) \sin \left(\frac{\eta}{2}-\zeta-u\right) h(u), \\
x_{2}(u) & =\sin \left(\frac{\eta}{2}+\zeta-u\right) \sin \left(\frac{\eta}{2}-\zeta-u\right) h(u), \\
x_{3}(u) & =\sin \left(\frac{\eta}{2}+\zeta-u\right) \sin \left(\frac{\eta}{2}-\zeta+u\right) h(u),
\end{aligned}
$$

where $\nu \neq 0$ and $\zeta(\sin 2 \zeta \neq 0)$ are arbitrary constants and $h(u) \not \equiv 0$ is an arbitrary function.

For the case (a2-2), we may take $\zeta=0$ or $\frac{1}{2} \pi$ because (A.23) is periodic in $\zeta$ with period $\pi$. For both the $\zeta$ values, Eq[2,6] implies $\sin (\eta-2 u) g(u)=($ constant $) \times \sin 2 u f(u)$. However Eq[1,3] implies this constant is zero, which contradicts $g(u) \not \equiv 0$. 
- Case (a3). From (A.17) we have $x_{1}(u)-x_{3}(u)=\sigma \sin (\eta-2 u) g(u)$, where $\sigma$ is a constant. From (A.23) we get $-\sin 2 \zeta \sin 2 u f(u)=\sigma \sin (\eta-2 u) g(u)$. Again two choices are possible:

$$
\begin{array}{ll}
(\text { a3 }-1) & \sin 2 \zeta \neq 0(\Rightarrow \sigma \neq 0), \\
(\text { a3-2 }) & \sin 2 \zeta=0(\Rightarrow \sigma=0) .
\end{array}
$$

For the case (a2-1), Eq[3,1] implies $\sin 4 \eta=0$ and then we can check that (3.1) is satisfied. Therefore we get a solution (note again: $\sin 4 \eta=0$ ),

$$
\begin{aligned}
\tilde{z}(u) & =\tilde{\nu} \sin 2 u h(u), \quad y_{1}(u)=y_{2}(u)=\tilde{y}_{1}(u)=\tilde{y}_{2}(u)=z(u)=0, \\
x_{1}(u) & =\sin \left(\frac{\eta}{2}+\zeta+u\right) \sin \left(\frac{\eta}{2}-\zeta-u\right) h(u), \\
x_{2}(u) & =\sin \left(\frac{\eta}{2}+\zeta-u\right) \sin \left(\frac{\eta}{2}-\zeta-u\right) h(u), \\
x_{3}(u) & =\sin \left(\frac{\eta}{2}+\zeta-u\right) \sin \left(\frac{\eta}{2}-\zeta+u\right) h(u),
\end{aligned}
$$

where $\tilde{\nu} \neq 0$ and $\zeta(\sin 2 \zeta \neq 0)$ are arbitrary constants and $h(u) \not \equiv 0$ is an arbitrary function. For the case $(\mathrm{a} 3-2), \mathrm{Eq}[6,2]$ implies $\sin (\eta-2 u) g(u)=($ constant $) \times \sin 2 u f(u)$. However Eq[3,1] implies this constant is zero, which contradicts $g(u) \not \equiv 0$.

IV.2. Case (b).

A.18) with $u^{\prime}=0$ implies that $X_{1}(u)+X_{2}(u)$ is a constant, and (A.18) with $u^{\prime}=\frac{1}{2} \pi$ implies that $X_{1}(u)-X_{2}(u)$ is also a constant. Therefore $X_{1}$ and $X_{2}$ are both constants, $X_{1}(u)=c_{1}, X_{2}(u)=c_{2}$ with $c_{1}^{2}-c_{2}^{2}+\nu \tilde{\nu}=0$. Similarly from (A.19) we have $X_{3}(u)=c_{3}$ with $c_{3}^{2}-c_{2}^{2}+\nu \tilde{\nu}=0$. We have two possibilities, $c_{3}=c_{1}$ and $c_{3}=-c_{1}$. We eliminate $\tilde{\nu}$ using $\tilde{\nu}=\left(c_{2}^{2}-c_{1}^{2}\right) / \nu$.

- Case $c_{3}=c_{1}$. Eq[2,6] implies $c_{1}=0$ and $\mathrm{Eq}[1,3]$ implies $c_{2}=0$. But this contradicts $\tilde{\nu} \neq 0$.

- Case $c_{3}=-c_{1}$. Eq[3,7] implies $c_{1}=0$ or $c_{2}=0$. For $c_{1}=0, \operatorname{Eq}[1,3]$ implies $c_{2}=0$ but this contradicts $\tilde{\nu} \neq 0$. For $c_{2}=0, \operatorname{Eq}[1,3]$ implies $c_{1}=0$ or $\sin 4 \eta=0$. The former contradicts $\tilde{\nu} \neq 0$. For the latter case we can check (3.1) is satisfied. Therefore we get a solution (for $\sin 4 \eta=0$ ),

$$
\begin{aligned}
& y_{1}(u)=y_{2}(u)=\tilde{y}_{1}(u)=\tilde{y}_{2}(u)=x_{2}(u)=0, \\
& x_{1}(u)=h(u), x_{3}(u)=-h(u), z(u)=\nu h(u), \tilde{z}(u)=-\frac{1}{\nu} h(u),
\end{aligned}
$$

where $\nu \neq 0$ is an arbitrary constant and $h(u) \not \equiv 0$ is an arbitrary function.

V. Case (ii), (iii)

First let us consider case (ii). Eq[6,2] implies $\tilde{\mu}^{2}=4 \lambda \tilde{\nu} \cos \eta$. Then we can check that (3.1) is satisfied. Therefore we get a solution,

$$
y_{1}(u)=y_{2}(u)=z(u)=0,
$$




$$
\begin{aligned}
& \tilde{y}_{1}(u)=\tilde{\mu} \sin \left(\zeta-\frac{\eta}{2}+u\right) g(u), \quad \tilde{y}_{2}(u)=\tilde{\mu} \sin \left(\zeta+\frac{\eta}{2}-u\right) g(u), \tilde{z}(u)=\tilde{\nu} \sin (\eta-2 u) g(u), \\
& x_{1}(u)=\frac{\tilde{\mu}^{2}}{2 \tilde{\nu} \cos \eta \sin 2 u} \sin \left(\frac{\eta}{2}+\zeta+u\right) \sin \left(\frac{\eta}{2}-\zeta-u\right) g(u), \\
& x_{2}(u)=\frac{\tilde{\mu}^{2}}{2 \tilde{\nu} \cos \eta \sin 2 u} \sin \left(\frac{\eta}{2}+\zeta-u\right) \sin \left(\frac{\eta}{2}-\zeta-u\right) g(u), \\
& x_{3}(u)=\frac{\tilde{\mu}^{2}}{2 \tilde{\nu} \cos \eta \sin 2 u} \sin \left(\frac{\eta}{2}+\zeta-u\right) \sin \left(\frac{\eta}{2}-\zeta+u\right) g(u),
\end{aligned}
$$

where $\zeta, \tilde{\mu}, \tilde{\nu}$ are arbitrary constant $(\tilde{\mu} \neq 0, \tilde{\nu} \neq 0)$ and $g(u) \not \equiv 0$ is an arbitrary function.

Case (iii) is treated similarly: Eq[2,6] implies $\mu^{2}=4 \lambda \nu \cos \eta$ and we get a solution,

$$
\begin{aligned}
& y_{1}(u)=\mu \sin \left(\zeta-\frac{\eta}{2}+u\right) g(u), \quad y_{2}(u)=\mu \sin \left(\zeta+\frac{\eta}{2}-u\right) g(u), \quad z(u)=\nu \sin (\eta-2 u) g(u), \\
& \tilde{y}_{1}(u)=\tilde{y}_{2}(u)=\tilde{z}(u)=0, \\
& x_{1}(u)=\frac{\mu^{2}}{2 \nu \cos \eta \sin 2 u} \sin \left(\frac{\eta}{2}+\zeta+u\right) \sin \left(\frac{\eta}{2}-\zeta-u\right) g(u), \\
& x_{2}(u)=\frac{\mu^{2}}{2 \nu \cos \eta \sin 2 u} \sin \left(\frac{\eta}{2}+\zeta-u\right) \sin \left(\frac{\eta}{2}-\zeta-u\right) g(u), \\
& x_{3}(u)=\frac{\mu^{2}}{2 \nu \cos \eta \sin 2 u} \sin \left(\frac{\eta}{2}+\zeta-u\right) \sin \left(\frac{\eta}{2}-\zeta+u\right) g(u),
\end{aligned}
$$

where $\zeta, \mu, \nu$ are arbitrary constant $(\mu \neq 0, \nu \neq 0)$ and $g(u) \not \equiv 0$ is an arbitrary function.

VI. Case (iv-a)

Eq[1,7] implies $\mu=0$ and Eq[7,1] implies $\tilde{\mu}=0$. But this contradicts the assumption. Therefore there are no solutions for this case.

VII. Case (iv-b)

$\mathrm{Eq}[2,4]$ implies

$$
\lambda=\frac{\mu^{3} \sin \left(\frac{\eta}{2}+\zeta\right) \sin \left(\frac{\eta}{2}-\zeta\right)+\tilde{\mu} \nu^{2} \sin \eta \sin 2 \eta}{4 \mu \nu \cos \eta \sin \left(\frac{\eta}{2}+\zeta\right) \sin \left(\frac{\eta}{2}-\zeta\right)} .
$$

Then we can check that (3.1) is satisfied. Therefore we get a solution,

$$
\begin{aligned}
& y_{1}(u)=\mu \sin \left(\zeta-\frac{\eta}{2}+u\right) g(u), \quad y_{2}(u)=\mu \sin \left(\zeta+\frac{\eta}{2}-u\right) g(u), \quad z(u)=\nu \sin (\eta-2 u) g(u), \\
& \tilde{y}_{1}(u)=\tilde{\mu} \sin \left(\zeta-\frac{\eta}{2}+u\right) g(u), \quad \tilde{y}_{2}(u)=\tilde{\mu} \sin \left(\zeta+\frac{\eta}{2}-u\right) g(u), \quad \tilde{z}(u)=\tilde{\nu} \sin (\eta-2 u) g(u), \\
& x_{1}(u)=\frac{\mu^{3} \sin \left(\frac{\eta}{2}+\zeta+u\right) \sin \left(\frac{\eta}{2}-\zeta-u\right)+\tilde{\mu} \nu^{2} \sin 2 \eta \sin (\eta-2 u)}{2 \mu \nu \cos \eta \sin 2 u} g(u), \\
& x_{2}(u)=\frac{\mu^{3} \sin \left(\frac{\eta}{2}+\zeta-u\right) \sin \left(\frac{\eta}{2}-\zeta-u\right)+2 \tilde{\mu} \nu^{2} \cos \eta \sin (\eta-2 u) \sin (\eta+2 u)}{2 \mu \nu \cos \eta \sin 2 u} g(u), \\
& x_{3}(u)=\frac{\mu^{3} \sin \left(\frac{\eta}{2}+\zeta-u\right) \sin \left(\frac{\eta}{2}-\zeta+u\right)+\tilde{\mu} \nu^{2} \sin 2 \eta \sin (\eta-2 u)}{2 \mu \nu \cos \eta \sin 2 u} g(u),
\end{aligned}
$$

where $\zeta, \mu, \tilde{\mu}, \nu$ are arbitrary constants $\left(\mu \neq 0, \tilde{\mu} \neq 0, \nu \neq 0, \tilde{\nu}=\tilde{\mu}^{2} \nu / \mu^{2}\right)$ and $g(u) \not \equiv 0$ is an arbitrary function. 
VIII. To summarize, we have obtained all the solutions of BYBE. (A.27), (A.29), (A.30) correspond to three solutions in Class (B). Other solutions (A.21), (A.22), (A.25), (A.31), (A.32), (A.34) can be expressed in one formula (3.3) (Class (A)). For example, (A.34) is obtained from (3.3) by replacing $\mu \rightarrow 2 \nu \mu^{-1} \cos \eta, \tilde{\mu} \rightarrow 2 \tilde{\mu} \nu \mu^{-2} \cos \eta$ and dividing by $2 \nu \mu^{-2} \cos \eta \sin 2 u$. (A.21) is obtained from (3.3) by setting $\tilde{\mu}=0$, dividing by $\mu^{2}$, and taking a limit $\mu \rightarrow \infty$. But this solution can not satisfy (3.5).

\section{References}

[1] I. Cherednik, Theor. Math. Phys. 61 (1984) 35.

[2] E.K. Sklyanin, J. Phys. A: Math. Gen. 21 (1988) 2375.

[3] S. Ghoshal and A.B. Zamolodchikov, Int. J. Mod. Phys. A9 (1994) 3841.

[4] J. Cardy, Nucl. Phys. B324 (1989) 581;

C.G. Callan, I.R. Klebanov, A.W.W. Ludwig and J.M. Maldacena, Nucl. Phys. B422 (1994) 417.

[5] T. Inami, S. Odake and Y.-Z. Zhang, Phys. Lett. B359 (1995) 118

C. Ahn and W.-M. Woo, preprint hep-th/9509056

[6] T. Inami, H. Konno and Y.-Z. Zhang, preprint YITP-95-16, hep-th/9512085.

[7] E. Corrigan, P.E. Dorey, R.H. Rietdijk and R. Sasaki, Phys. Lett. B333 (1994) 83;

S. Penati, A. Refolli and D. Zanon, preprint IFUM-522-FT, hep-th/9512174.

[8] H.J. de Vega and A. González-Ruiz, J. Phys. A: Math. Gen. 26 (1993) L519.

[9] M. Jimbo, R. Kedem, T. Kojima, H. Konno and T. Miwa, Nucl. Phys. B441 (1995) 437.

[10] B.-Y. Hou and R.-H. Yue, Phys. Lett. A183 (1993) 169;

T. Inami and H. Konno, J. Phys. A: Math. Gen. 27 (1994) L913.

[11] L. Mezincescu, R.I. Nepomechie and V. Rittenberg, Phys. Lett. A147 (1990) 70.

[12] A.B. Zamolodchikov and V.A. Fateev, Sov. J. Nucl. Phys. 32 (1980) 298.

[13] P.P. Kulish, N.Yu. Reshetikhin and E.K. Sklyanin, Lett. Math. Phys. 5 (1981) 393. 